\title{
Meshless Method of Lines for Numerical Solution of Kawahara Type Equations
}

\author{
Nagina Bibi, Syed Ikram Abbas Tirmizi*, Sirajul Haq \\ Faculty of Engineering Sciences, Ghulam Ishaq Khan Institute, Topi, Pakistan \\ E-mail: tirmizi@giki.edu.pk \\ Received Feburary 22, 2011; revised March 26, 2011; accepted March 30, 2011
}

\begin{abstract}
In this work, an algorithm based on method of lines coupled with radial basis functions namely meshless method of lines (MMOL) is presented for the numerical solution of Kawahara, modified Kawahara and KdV Kawahara equations. The motion of a single solitary wave, interaction of two and three solitons and the phenomena of wave generation is discussed. The results are compared with the exact solution and with the results in the relevant literature to show the efficiency of the method.
\end{abstract}

Keywords: Kawahara Type Equations, Meshless Method of Lines (MMOL), Radial Basis Functions (RBFs)

\section{Introduction}

In this paper we study numerical solution of Kawahara equation, Modified Kawahara equation and KdV-Kawahara equation respectively given as:

$$
\begin{gathered}
u_{t}+u u_{x}+u_{x x x}-u_{x x x x}=0, \quad a \leq x \leq b, \quad t \geq 0 \\
u_{t}+u^{2} u_{x}+u_{x x x}-u_{x x x x}=0, \quad a \leq x \leq b, \quad t \geq 0 \\
u_{t}+u_{x}+u u_{x}+u_{x x x}-u_{x x x x x}=0, \quad a \leq x \leq b, \quad t \geq 0
\end{gathered}
$$

A variety of physical phenomena like, magneto acoustic waves in plasma [1], shallow water waves with surface tension [2] and capillary gravity water waves [3], are described by Kawahara Equation (1) and modified Kawahara Equation (2). KdV-Kawahara Equation (3) is used to describe the one dimensional evolution of small but finite amplitude long waves in various problems in fluid dynamics. This equation is a specific form of Benney-Lin equation $[4,5]$.

Different analytic and numerical methods including the tanh-function method [6], Adomian decomposition method [7], sine-cosine method [8], variational iteration method, homotopy perturbation method [9], Crank-Nicolson Differential quadrature algorithms [10], Predictor corrector methods [11], Dual-Petrov Galerkin method [12] and RBF collocation method [13] have been proposed for solving the Kawahara type equations.

We shall use method of lines approach [14,15] using RBFs for numerical solution of the above problems using radial basis functions. The method of lines [16] is pow- erful and comprehensive approach for solving time dependent partial differential equations (PDEs). This method involves two steps, first the approximation of spatial derivatives converting the PDEs to a system of ordinary differential equations (ODEs) and in the second step the resulting system of ODEs is integrated in time. One of the salient features of the MOL is the use of existing and well established numerical methods for ODEs. Coupling method of lines with radial basis functions makes it very simple by getting rid of mesh generation as compared to conventional mesh based methods. In 1990 Kansa [17, 18] for the first time used radial basis functions for numerical solution of PDEs. Other researchers like Fornberg et al. [19], Hon and Wu [20], Franke and Schaback [21], Wong et al. [22], Chen and Tanaka [23] also contributed a lot in this area. In order to solve the resultant collocation method Fasshauer modified the Kansa's method to a Hermite type collocation method [24]. For nonlinear time dependent PDEs see also [25,26]. In 1971 Hardy [27], developed MQ to approximate geogra- phical surfaces. In Franke's [28] review paper, the MQ was ranked the best in some thirty scattered data interpolation methods. For solving PDEs the convergence proofs in applying the RBF's is given by Wu [29]. The accuracy of RBFs methods depends on the choice of a parameter called shape parameter involved in infinitely smooth RBFs like, Multiquadric (MQ), Gaussian (GA), Inverse multiquadric (IMQ) radial basis functions. Tarwater [30] found that the Root Mean Square of error decreases up to certain limit and then increases rapidly when $c \rightarrow \infty$. 
Golberg, Chen, and Karur [31] and Hickernell and Hon [32] applied the technique of cross validation to obtain an optimal value of the shape parameter.

However the methods based on globally supported radial basis functions (GSRBFs) approach face the problem of ill-conditioning of the dense interpolation matrix. In order to overcome these difficulties several alternatives including domain decomposition [33], preconditioning [34] and use of compactly supported RBF [35] have been introduced. Another useful approach in this regard is locally supported RBFs instead of globally supported RBFs. The readers are recommended to visit $\mathrm{Wu}$ and Liu [36], C. K. Lie et al. [37], R. Vertnik and Bozidar Sarler [38]. It is worth mentioning that global, infinitely differentiable RBFs typically interpolate smooth data with spectral accuracy [39-42] and the shape parameter can be adjusted with the number of centers in order to produce a interpolation matrix which is well conditioned enough to be inverted in finite precision arithmetic [43].The globally supported RBFs were used early on and for the problems whose size does not exceed 400-500 data points. These methods should still be the method of choice [44].

This paper is organized in three sections. In Section 2 the numerical scheme is explained and Section 3 contains the numerical examples for the justification of the method and we conclude in Section 4.

\section{Numerical Scheme}

For implementation of numerical method, we consider the Kawahara Equation (1) with the following initial and boundary conditions

$$
\begin{gathered}
u(x, 0)=f(x), a \leq x \leq b, \\
u(a, t)=g_{1}(t), u(b, t)=g_{2}(t)
\end{gathered}
$$

To apply meshless method of lines, we first use radial basis functions to approximate space derivatives. The problem domain $[a, b]$ is divided into nodes $x_{i}, i=1,2, \cdots, N$. Out of these points $x_{i}, i=2, \cdots, N-1$ are interior points and $x_{i}, i=1$ and $N$ are the boundary points.

The approximate solution of $u(x, t)$ is given by

$$
u(x, t)=u^{n}(x, t)=\sum_{j=1}^{N} \lambda_{j}^{n}(t) \psi\left(x_{j}\right)=\Psi^{\mathrm{T}}(x) \lambda,
$$

where

$$
\Psi^{\mathrm{T}}(x)=\left[\psi_{1}(x), \psi_{2}(x), \cdots, \psi_{N}(x)\right]^{\mathrm{T}}
$$

We have used the following RBFs

$$
\begin{gathered}
\psi\left(x_{j}\right)=\sqrt{\left(x-x_{j}\right)^{2}+c^{2}}, M Q \\
\psi\left(x_{j}\right)=\exp \left(-c\left(x-x_{j}\right)^{2}\right), G A
\end{gathered}
$$

$$
\psi\left(x_{j}\right)=\frac{1}{\sqrt{\left(x-x_{j}\right)^{2}+c^{2}}}, I M Q
$$

where $j=1,2, \cdots, N$ and $c$ being shape parameter.

The Equation (6) in the matrix form is

$$
\boldsymbol{u}=\boldsymbol{A \lambda}
$$

where

$$
\boldsymbol{u}=\left[u_{1}(t), u_{2}(t), \cdots, u_{N}(t)\right]^{\mathrm{T}}, \lambda=\left[\lambda_{1}, \lambda_{2}, \cdots, \lambda_{N}\right]^{\mathrm{T}}
$$

and

$$
\boldsymbol{A}=\left[\begin{array}{c}
\boldsymbol{\Psi}^{\mathrm{T}}\left(x_{1}\right) \\
\boldsymbol{\Psi}^{\mathrm{T}}\left(x_{2}\right) \\
\cdots \\
\Psi^{\mathrm{T}}\left(x_{N}\right)
\end{array}\right]=\left[\begin{array}{cccc}
\psi_{1}\left(x_{1}\right) & \psi_{2}\left(x_{1}\right) & \ldots & \psi_{N}\left(x_{1}\right) \\
\psi_{1}\left(x_{2}\right) & \psi_{2}\left(x_{2}\right) & \cdots & \psi_{N}\left(x_{2}\right) \\
\cdot & \cdot & \cdot & \cdot \\
\cdot & \cdot & \cdot & \cdot \\
\cdot & \cdot & & \cdot \\
\psi_{1}\left(x_{N}\right) & \psi_{2}\left(x_{N}\right) & \cdots & \psi_{N}\left(x_{N}\right)
\end{array}\right]
$$

Using Equation (7) in Equation (6) it follows that

$$
u(x, t)=\Psi^{\mathrm{T}}(x) \boldsymbol{A}^{-1} \boldsymbol{u}=\boldsymbol{S}(x) \boldsymbol{u},
$$

where $\boldsymbol{S}(x)=\Psi^{\mathrm{T}}(x) \boldsymbol{A}^{-1}$

Applying Equation (8) to Equation (1), and collocating at each node $x_{i}$, we get system of first order ODEs

$$
\begin{aligned}
& \frac{\mathrm{d} u_{i}}{\mathrm{~d} t}+u_{i}\left(\boldsymbol{S}_{x}\left(x_{i}\right) \boldsymbol{u}\right)+\left(\boldsymbol{S}_{x x x}\left(x_{i}\right) \boldsymbol{u}\right)-\left(\boldsymbol{S}_{x x x x}\left(x_{i}\right) \boldsymbol{u}\right)=0, \\
& i=1,2, \cdots, N
\end{aligned}
$$

where $u_{i}(t)=u_{i}$

$$
\begin{aligned}
& \boldsymbol{S}_{x}\left(x_{i}\right)=\left[S_{1 x}\left(x_{i}\right), S_{2 x}\left(x_{i}\right), \cdots, S_{N x}\left(x_{i}\right)\right], i=1,2, \cdots, N \\
& \boldsymbol{S}_{j x}\left(x_{i}\right)=\frac{\partial}{\partial x} S_{j}\left(x_{i}\right), i, j=1,2, \cdots, N
\end{aligned}
$$

In the similar fashion

$$
\begin{gathered}
\boldsymbol{S}_{x x x}\left(x_{i}\right)=\left[S_{1 x x x}\left(x_{i}\right), S_{2 x x x}\left(x_{i}\right), \cdots, S_{N x x x}\left(x_{i}\right)\right], \\
i=1,2, \cdots, N \\
S_{j x x x}\left(x_{i}\right)=\frac{\partial^{3}}{\partial x^{3}} S_{j}\left(x_{i}\right), i, j=1,2, \cdots, N \\
\boldsymbol{S}_{x x x x x}\left(x_{i}\right)=\left[S_{1 x x x x x}\left(x_{i}\right), S_{2 x x x x}\left(x_{i}\right), \cdots, S_{N x x x x x}\left(x_{i}\right)\right], \\
i=1,2, \cdots, N \\
S_{j x x x x x}\left(x_{i}\right)=\frac{\partial^{5}}{\partial x^{5}} S_{j}\left(x_{i}\right), i, j=1,2, \cdots, N
\end{gathered}
$$

In order to write the above system of equations in terms of column vectors, let

$$
\begin{aligned}
& \boldsymbol{U}=\left[\begin{array}{llllll}
u_{1} & u_{2} & u_{3} & \ldots & u_{N-1} & u_{N}
\end{array}\right]^{\mathrm{T}}, \\
& \boldsymbol{S}_{x}=\left[S_{j x}\left(x_{i}\right)\right]_{N \times N},
\end{aligned}
$$




$$
\begin{aligned}
& \boldsymbol{S}_{x x x}=\left[S_{j x x x}\left(x_{i}\right)\right]_{N \times N}, \\
& \boldsymbol{S}_{x x x x x}=\left[S_{j x x x x x}\left(x_{i}\right)\right]_{N \times N},
\end{aligned}
$$

Equation (9) then can be written as follows

$$
\frac{\mathrm{d} \boldsymbol{U}}{\mathrm{d} t}+\boldsymbol{U} *\left(\boldsymbol{S}_{x} \boldsymbol{U}\right)+\left(\boldsymbol{S}_{x x x} \boldsymbol{U}\right)-\left(\boldsymbol{S}_{x x x x x} \boldsymbol{U}\right)=\mathbf{0}
$$

Rewriting Equation (10) as

$$
\frac{\mathrm{d} \boldsymbol{U}}{\mathrm{d} t}=G(\boldsymbol{U})
$$

where

$$
G(\boldsymbol{U})=-\boldsymbol{U} *\left(\boldsymbol{S}_{x} \boldsymbol{U}\right)-\left(\boldsymbol{S}_{x x x} \boldsymbol{U}\right)+\left(\boldsymbol{S}_{x x x x x} \boldsymbol{U}\right) \text { and the sy- }
$$

mbol (*) denotes component by component multiplication of two vectors.

The initial condition is

$$
\boldsymbol{U}\left(t_{0}\right)=\left[u^{0}\left(x_{1}\right), u^{0}\left(x_{2}\right), \cdots, u^{0}\left(x_{N}\right)\right]
$$

From the boundary conditions described in (5) we get

$$
u_{1}(t)=g_{1}(t) \text { and } u_{N}(t)=g_{2}(t)
$$

Now we use classical fourth order Runge-Kutta scheme to solve Equations (11)-(13), namely

$$
\left\{\begin{array}{l}
\boldsymbol{U}^{n+1}=\boldsymbol{U}^{n}+\frac{\Delta t\left(K_{1}+2\left(K_{2}+K_{3}\right)+K_{4}\right)}{6} \\
K_{1}=G\left(\boldsymbol{U}^{n}\right), K_{2}=G\left(\boldsymbol{U}^{n}+\frac{\Delta t}{2} K_{1}\right) \\
K_{3}=G\left(\boldsymbol{U}^{n}+\frac{\Delta t}{2} K_{2}\right), K_{4}=G\left(\boldsymbol{U}^{n}+\Delta t K_{3}\right)
\end{array}\right.
$$

The RK4 scheme does not face problem of stability as long as the time step $\Delta t$ is chosen sufficiently small (see Collatz [45]) which is so in our case. The rule of thumb in selection of the time step $\Delta t$ for stability is as follows [46]: "The method of lines is stable if the eigenvalues of the (linearized) spatial discretization operator, scaled by time step $\Delta t$, lie in the stability region of the timediscretization operator". So the method is stable if all the eigenvalues $\left(\alpha_{j}, j=1,2, \cdots, N\right)$ of the Jacobian matrix in (11) lie inside the stability region $R\left(\right.$ i.e. $\left.-2.78<\Delta t \alpha_{j}<0\right)$ of RK4 scheme. For further details regarding stability of Runge-Kutta fourth order method, see Lambert [47] and Jain [48]. As far as the selection of the shape parameter $c$ is concerned in this work, first we have to find an interval for $c$ in which matrix $\boldsymbol{A}$ of radial basis functions is invertible and then select a value from that interval which gives us the most accurate results.

\section{Numerical Application}

In this section the numerical results for Kawahara, modified Kawahara and KdV Kawahara equations are pre- sented. The root mean square norm and maximum error norms are calculated by the formulas

$$
\begin{gathered}
L_{2}=\left|u_{\text {ex }}-u_{\text {num }}\right|^{2}=\sum_{j=1}^{N} \Delta x\left|\left(u_{\text {ex }}(j)-u_{\text {num }}(j)\right)^{2}\right| \\
L_{\infty}=\left|u_{\text {ex }}-u_{\text {num }}\right|_{\infty}=\max _{j}\left|u_{e x}(j)-u_{\text {num }}(j)\right|
\end{gathered}
$$

For Kawahara equation the lowest three conserved quantities, defined in [49] are also calculated using

$$
\begin{aligned}
& I_{1}=\int_{-\infty}^{\infty} u \mathrm{~d} x, I_{2}=\int_{-\infty}^{\infty} \frac{1}{2} u^{2} \mathrm{~d} x, \\
& I_{3}=\int_{-\infty}^{\infty}\left(\frac{13}{8} \delta u_{x}^{2}-\frac{105}{96} \lambda^{2} u^{3}+\frac{1}{2} u_{x x}^{2}\right) \mathrm{d} x
\end{aligned}
$$

Example 3.1

Consider the Kawahara equation

$$
u_{t}+u u_{x}+u_{x x x}-u_{x x x x}=0
$$

with the following initial and boundary conditions

$$
\begin{gathered}
u(x, 0)=\frac{105}{169} \operatorname{sech}^{4}\left(k\left(x-x_{o}\right)\right) \\
u(a, t)=0 ; u(b, t)=0
\end{gathered}
$$

The exact solution given in [10] is,

$$
u(x, t)=\frac{105}{169} \operatorname{sech}^{4}\left(k\left(x-\frac{36}{169} t-x_{o}\right)\right)
$$

where $k=\frac{1}{2} \sqrt{\frac{1}{13}}$

For numerical computation we take $[a, b]=[-20,30]$, $x_{o}=2, \Delta x=1, N=51$. The simulation is carried out up to time $t=25 . L_{2}$ and $L_{\infty}$ norms are calculated at $t=0,5$, 15 and 25, using MQ and GA radial basis functions with the value of shape parameter found to be in neighborhood of 4 as shown in Figure 1 and similarly for GA the value is found to be in neighborhood of 0.3 . We have searched the optimal value of the shape parameter by plotting maximum error verses shape parameter with step 0.01. The three conserved quantities are also shown in the Table 1.The amplitudes and peak position of the solitary waves are also calculated. The results with present method using MQ are better than the polynomial based differential quadrature (PDQ) method [10] and are very close to cosine expansion based differential quadrature (CDQ) method [10]. While the results obtained by GA are better than both methods mentioned in [10]. In Figure 2 the forward motion of the solitary wave in comparison with exact solution (19) at different time levels is shown.

The Point wise rate of convergence in space is calculated using the following formula: 
Table 1. Results for Kawahara equation in comparison with [10].

\begin{tabular}{|c|c|c|c|c|c|c|c|c|c|}
\hline Method & Time & $L_{2} \times 10^{3}$ & $L_{\infty} \times 10^{3}$ & $I_{1}$ & $I_{2}$ & $I_{3}$ & Height & $\begin{array}{c}\text { Peak } \\
\text { Position }\end{array}$ & $\begin{array}{c}\text { CPU } \\
\text { time(s) }\end{array}$ \\
\hline \multirow[t]{5}{*}{$\mathrm{MQ}(c=4.3)$} & 0 & 0 & 0 & 5.97359 & 1.27250 & -0.16458 & 0.62130 & 2 & \\
\hline & 5 & 0.09468 & 0.04669 & 5.97348 & 1.27250 & -0.16458 & 0.62119 & 3 & 0.094 \\
\hline & 15 & 0.15362 & 0.05939 & 5.97343 & 1.27250 & -0.16458 & 0.62038 & 5 & 0.188 \\
\hline & 25 & 0.16818 & 0.04660 & 5.97355 & 1.27250 & -0.16458 & 0.61880 & 7 & 0.328 \\
\hline & 0 & 0 & 0 & 5.973599 & 1.272502 & -0.16458 & 0.621301 & 2 & \\
\hline \multirow[t]{4}{*}{$\mathrm{GA}(c=0.27)$} & 5 & 0.10075 & 0.034297 & 5.973662 & 1.272502 & -0.16458 & 0.621201 & 3 & 0.171 \\
\hline & 15 & 0.10113 & 0.03830 & 5.973675 & 1.272502 & -0.16458 & 0.620382 & 5 & 0.172 \\
\hline & 25 & 0.13160 & 0.03990 & 5.973532 & 1.272502 & -0.16458 & 0.618802 & 7 & 0.281 \\
\hline & 0 & 0 & 0 & 5.97357 & 1.27250 & -0.16458 & 0.62130 & 2 & \\
\hline \multirow[t]{3}{*}{$\mathrm{PDQ}[10](\Delta t=0.1)$} & 5 & 1.986 & 0.921 & 5.97060 & 1.27250 & -0.16458 & 0.62102 & 3 & \\
\hline & 15 & 2.543 & 1.045 & 5.97014 & 1.27250 & -0.16458 & 0.62047 & 5 & \\
\hline & 25 & 2.851 & 0.863 & 5.97353 & 1.27250 & -0.16457 & 0.61872 & 7 & \\
\hline \multirow[t]{4}{*}{ CDQ[10] } & 0 & 0 & 0 & 5.97357 & 1.27250 & -0.16458 & 0.62130 & 2 & \\
\hline & 5 & 0.151 & 0.043 & 5.97372 & 1.27250 & -0.16458 & 0.62122 & 3 & \\
\hline & 15 & 0.156 & 0.049 & 5.97364 & 1.27250 & -0.16458 & 0.62037 & 5 & \\
\hline & 25 & 0.159 & 0.076 & 5.97350 & 1.27250 & -0.16458 & 0.61877 & 7 & \\
\hline
\end{tabular}

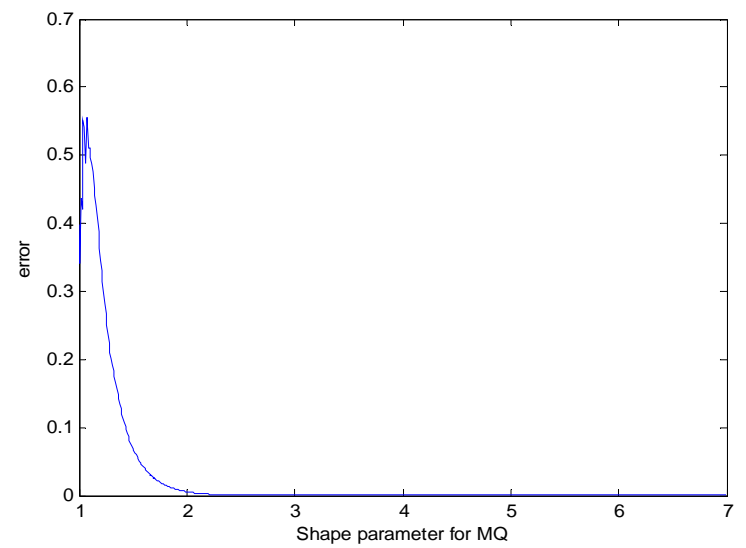

Figure 1. Error verses shape parameter c for Example 3.1.

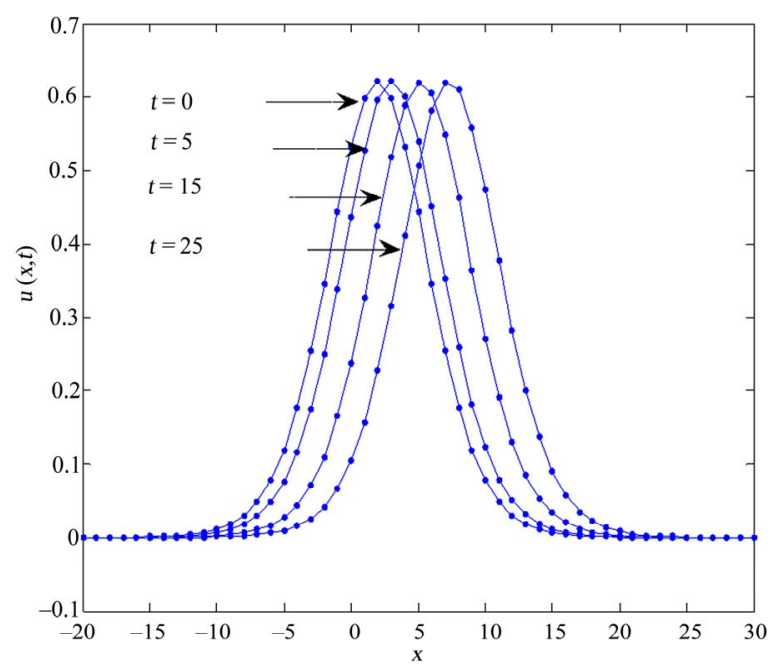

Figure 2. Travelling wave solution of Kawahara equation (solid lines showing numerical solution and dot (.) showing exact solution.

$$
\frac{\log _{10}\left(\left\|u-U_{h_{i}}\right\| /\left\|u-U_{h_{i+1}}\right\|\right)}{\log _{10}\left(h_{i} / h_{i+1}\right)}
$$

where $u$ and $U_{h_{i}}$ represents the exact solution and the numerical solution respectively and $h_{i}$ is spatial step size. We calculate spatial rate of convergence by keeping time step $\Delta t=0.001$ fixed and varying the number of collocation points $(F=20,40,80)$. From the Table 2 we can see that the order of convergence decreases with the smaller spatial step size. In all numerical examples we have used MQ and GA in order to calculate order of convergence.

Example 3.2

Consider the modified Kawahara equation

$$
u_{t}+u^{2} u_{x}+u_{x x x}-u_{x x x x}=0
$$

with the following initial and boundary conditions

$$
\begin{gathered}
u(x, 0)=D \operatorname{sech}^{2}(k(x)) \\
u(a, t)=D \operatorname{sech}^{2}(k(a-B t)) ; \\
u(b, t)=D \operatorname{sech}^{2}(k(b-B t))
\end{gathered}
$$

The above conditions are extracted from the exact solution given in [50],

$$
u(x, t)=D \operatorname{sech}^{2}(k(x-B t))
$$

where $D=-\frac{3}{\sqrt{10}}, k=\frac{1}{2} \sqrt{\frac{1}{5}}, B=\frac{4}{25}$

The calculation is carried out by taking $[a, b]=[-30,30]$ with $x=1$.We use MQ, GA and IMQ radial basis with shape parameter found to be in the neighborhood of 3 for MQ as shown in Figure 3 and for GA and IMQ it is in the neighborhood of 0.5 and 0.0001 . The $L_{2}$ and $L_{\infty}$ norms calculated at $t=0,5,15,25$ are shown in Table 3 . 
Table 2. Spatial rate of convergence at for Example 3.1, $t=25$.

\begin{tabular}{ccccc}
\hline$N$ & $L_{\infty}$ & order & $L_{2}$ & Order \\
\hline MQ & & & & \\
20 & $2.81001 \times 10^{-2}$ & & $1.01808 \times 10^{-1}$ & \\
40 & $5.07036 \times 10^{-5}$ & 9.1143 & $1.67092 \times 10^{-4}$ & 9.2509 \\
80 & $3.56316 \times 10^{-5}$ & 0.5089 & $1.28499 \times 10^{-4}$ & 0.3788 \\
GA & & & & \\
20 & $1.49094 \times 10^{-2}$ & & $5.38775 \times 10^{-2}$ & \\
40 & $5.01268 \times 10^{-5}$ & 8.2164 & $1.35443 \times 10^{-4}$ & 8.6358 \\
80 & $4.77379 \times 10^{-5}$ & 0.0704 & $1.25773 \times 10^{-4}$ & 0.1068 \\
\hline
\end{tabular}

Table 3. Results for Modified Kawahara equation.

\begin{tabular}{|c|c|c|c|c|c|c|}
\hline method & time & $L_{\infty}$ & $L_{2}$ & $\overline{I_{1}}$ & $I_{2}$ & CPU time(s) \\
\hline \multirow[t]{4}{*}{$\mathrm{MQ}(c=2.9)$} & 0 & 0 & 0 & -8.48525 & 2.68328 & \\
\hline & 5 & $6.1995 \times 10^{-5}$ & $1.7896 \times 10^{-4}$ & -8.48524 & 2.683176 & 0.187 \\
\hline & 15 & $1.0717 \times 10^{-4}$ & $2.7337 \times 10^{-4}$ & -8.48487 & 2.68296 & 0.313 \\
\hline & 25 & $1.2130 \times 10^{-4}$ & $3.4855 \times 10^{-4}$ & -8.48464 & 2.68275 & 0.453 \\
\hline \multicolumn{7}{|l|}{$\mathrm{GA}(c=0.43)$} \\
\hline & 0 & 0 & 0 & -8.48525 & 2.68328 & \\
\hline & 5 & $5.3996 \times 10^{-5}$ & $1.0804 \times 10^{-4}$ & -8.48510 & 2.68317 & 0.171 \\
\hline & 15 & $8.6124 \times 10^{-5}$ & $1.9575 \times 10^{-4}$ & -8.48472 & 2.68296 & 0.313 \\
\hline \multirow[t]{5}{*}{$\operatorname{IMQ}(c=0.0001)$} & 25 & $7.8371 \times 10^{-5}$ & $2.5257 \times 10^{-4}$ & -8.48442 & 2.68275 & 0.543 \\
\hline & 0 & 0 & 0 & -8.48525 & 2.683281 & \\
\hline & 5 & $1.80659 \times 10^{-1}$ & $5.2570 \times 10^{-1}$ & -8.48525 & 2.683281 & 0.203 \\
\hline & 15 & $5.18281 \times 10^{-1}$ & $5.2819 \times 10^{-1}$ & -8.48134 & 2.683307 & 0.343 \\
\hline & 25 & $7.63711 \times 10^{-1}$ & $2.2681 \times 10^{0}$ & -8.47434 & 2.683351 & 0.469 \\
\hline
\end{tabular}

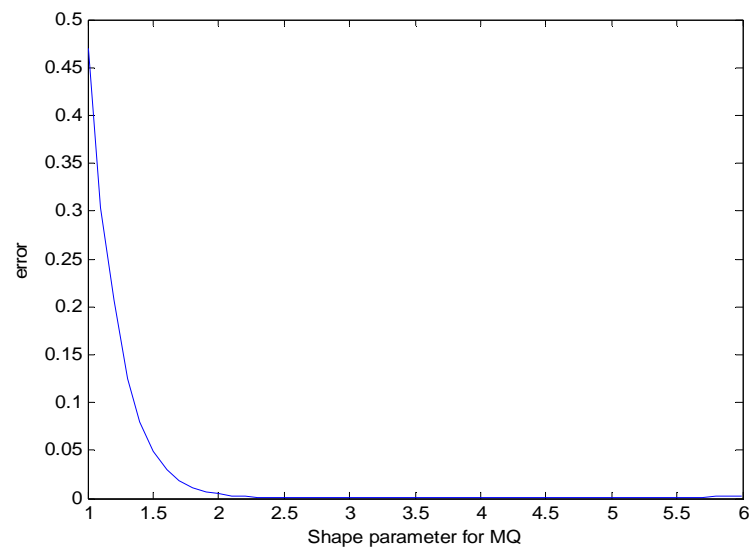

Figure 3. Error verses shape parameter c for Example 3.2.

GA and MQ are showing better accuracy than IMQ. The order of convergence in space decreases with increasing $N$ as shown in Table 4 . The solitary wave profile at different time levels in comparison with the exact solution is shown in Figure 4.

Example 3.3

Consider the KdV-Kawahara equation

$$
u_{t}+u u_{x}+u_{x}+u_{x x x}-u_{x x x x x}=0
$$

with the following initial and boundary conditions

$$
\begin{gathered}
u(x, 0)=\frac{105}{169} \operatorname{sech}^{4}\left(\frac{1}{2 \sqrt{13}}\left(x-x_{o}\right)\right) \\
u(x, a)=\frac{105}{169} \operatorname{sech}^{4}\left(\frac{1}{2 \sqrt{13}}\left(a-\frac{205}{169} t-x_{o}\right)\right)
\end{gathered}
$$

$$
u(x, b)=\frac{105}{169} \operatorname{sech}^{4}\left(\frac{1}{2 \sqrt{13}}\left(b-\frac{205}{169} t-x_{o}\right)\right)
$$

initial condition and boundary conditions are extracted from the exact solution given in [51].

$$
u(x, t)=\frac{105}{169} \operatorname{sech}^{4}\left(\frac{1}{2 \sqrt{13}}\left(x-\frac{205}{169} t-x_{o}\right)\right)
$$

The calculation is carried out by taking $[a, b]=[0,200]$ with $\Delta x=1$. The discrete root mean square error norm $L_{2}$ and maximum error norm $L_{\infty}$ are calculated using MQ, GA and IMQ for time $t=1$ up to 5. From the results shown in Table 5 we can see that the both MQ and GA are showing very good agreement with the exact solution. Shape parameter verses error plot for MQ is shown in Figure 5. The spatial rate of convergence is shown in Table 6. The order of convergence decreases by increasing collocation points for a fixed time step $\Delta t=$ 0.001 . The forward movement of the solitary wave at different time levels in comparison with the exact solution (26) is shown in Figure 6, same as in [52].

\section{Example 3.4}

Considering Equation (1) for interaction of two positive solitary waves with the following initial condition

$$
u(x, 0)=\sum_{i=1}^{2} A_{i}^{2} \operatorname{sech}^{4}\left(\frac{\sqrt{\sigma A_{i}}}{4}\left(x-x_{i}\right)\right) .
$$

We solve the problem by using MQ and GA RBFs taking $[a, b]=[-50,100]$ with $N=201$. The calculation is carried 
Table 4. Spatial rate of convergence at for Example 3.2, $t=5$.

\begin{tabular}{ccccc}
\hline$N$ & $L_{\infty}$ & order & $L_{2}$ & order \\
\hline MQ & & & $5.20872 \times 10^{-1}$ & \\
24 & $2.56860 \times 10^{-1}$ & & $2.65141 \times 10^{-3}$ & 7.6180 \\
48 & $1.49043 \times 10^{-3}$ & 7.4291 & $5.67016 \times 10^{-5}$ & 5.5472 \\
96 & $2.42285 \times 10^{-5}$ & 5.9428 & & \\
GA & & & $9.95424 \times 10^{-2}$ & 6.7583 \\
24 & $3.47477 \times 10^{-2}$ & & $9.19460 \times 10^{-4}$ & 4.0723 \\
48 & $5.00876 \times 10^{-4}$ & 6.1163 & $5.46571 \times 10^{-5}$ & \\
96 & $2.17409 \times 10^{-5}$ & 4.5259 & & \\
\hline
\end{tabular}

Table 5. Results for KdV Kawahara equation at $\boldsymbol{t}=\mathbf{5}$.

\begin{tabular}{ccccccc}
\hline method & $L_{\infty}$ & \multicolumn{1}{c}{$L_{2}$} & $I_{1}$ & $I_{2}$ & Amplitude & CPU time(s) \\
\hline $\mathrm{MQ}(c=2.6)$ & $1.0977 \times 10^{-4}$ & $3.7697 \times 10^{-4}$ & 5.97359 & 1.27250 & 0.621241 & 3.266 \\
$\mathrm{GA}(c=0.2)$ & $4.7924 \times 10^{-6}$ & $1.8465 \times 10^{-5}$ & 5.97368 & 1.27250 & 0.621200 & 3.390 \\
$\mathrm{IMQ}(c=0.0001)$ & $5.0111 \times 10^{-1}$ & $1.5920 \times 10^{0}$ & 5.95790 & 1.27250 & 0.619041 & 3.641 \\
\hline
\end{tabular}

Table 6. Spatial rate of convergence at for Example 3.3, $t=5$.

\begin{tabular}{ccccc}
\hline$N$ & $L_{\infty}$ & order & $L_{2}$ & order \\
\hline MQ & & & & \\
40 & $3.12550 \times 10^{-2}$ & 4.2648 & $4.60613 \times 10^{-3}$ & 4.8553 \\
80 & $1.62584 \times 10^{-3}$ & 2.1471 & $3.73104 \times 10^{-4}$ & 2.7242 \\
160 & $1.56826 \times 10^{-4}$ & & & \\
GA & & & $1.1620 \times 10^{-2}$ & 4.7639 \\
40 & $2.6983 \times 10^{-3}$ & 4.1971 & $3.2768 \times 10^{-3}$ & 3.7341 \\
80 & $1.4710 \times 10^{-4}$ & 3.5749 & $2.4624 \times 10^{-4}$ & \\
160 & $1.2344 \times 10^{-5}$ & & & \\
\hline
\end{tabular}

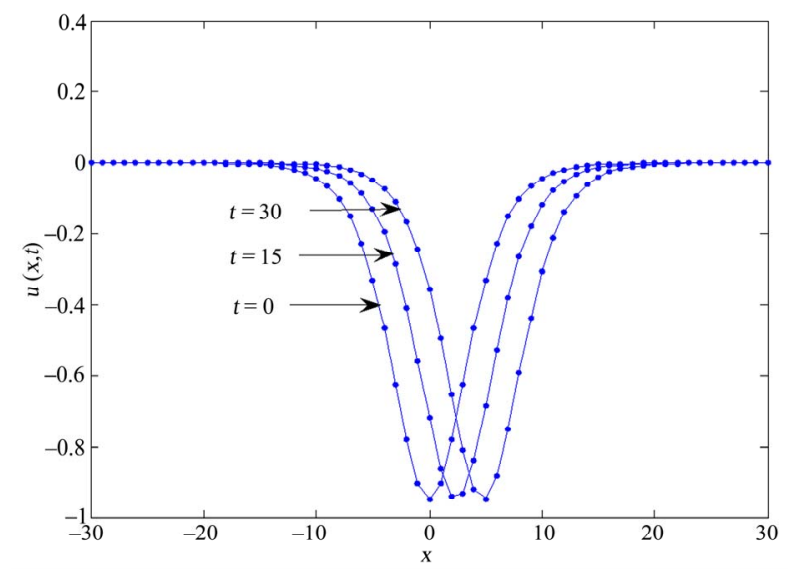

Figure 4. Solitary wave solution of modified Kawahara equation in comparison with exact solution (solid lines show numerical solution and dot (.) showing exact solution).

out up to time $t=50$ by taking time step $\Delta t=0.001$. For our numerical calculations the values of the parameters involved in above equation are chosen as:

$$
x_{1}=0, x_{2}=20, \sigma=\frac{4}{\sqrt{105}}, A_{1}=\frac{10}{\sigma}, A_{2}=\frac{8}{\sigma}
$$

The two solitary waves propagate towards right as the time progresses. The process of interaction is shown in Figure 7. During this process the larger wave catches up the smaller one and then the both waves separate from each other maintaining their original shape. From Table 7 , we can see that the invariants of motion remain almost conserved as time increases. The variation in the three

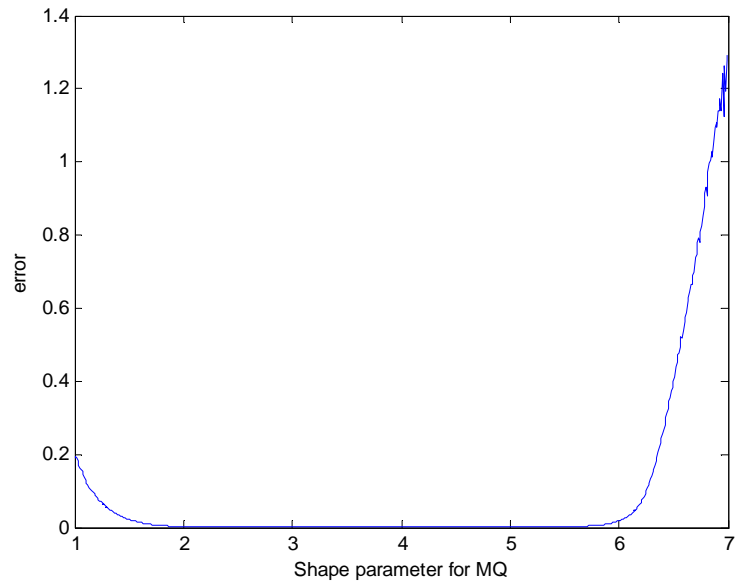

Figure 5. Error verses shape parameteric for Example 3.3.

conserved quantities is found to be in the range:

MQ $40.50925 \leq I_{1} \leq 40.48389$,

$45.83614 \leq I_{2} \leq 45.85093,-32.37219 \leq I_{3} \leq-32.15991$,

GA $40.50925 \leq I_{1} \leq 40.41284$,

$45.83614 \leq I_{2} \leq 45.84364,-32.37218 \leq I_{3} \leq-32.14082$.

\section{Example 3.5}

For interaction of three solitary waves we consider Equation (1) with the initial condition

$$
u(x, 0)=\sum_{i=1}^{3} A_{i}^{2} \operatorname{sech}^{4}\left(\frac{\sqrt{\sigma A_{i}}}{4}\left(x-x_{i}\right)\right)
$$




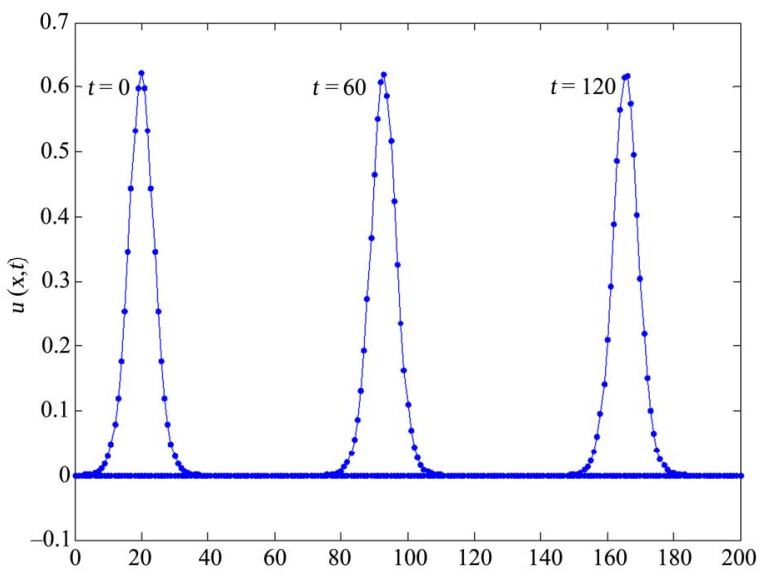

Figure 6. Solitary wave motion of KdV Kawahara equation (solid lines show numerical solution and dot (.) showing exact solution).

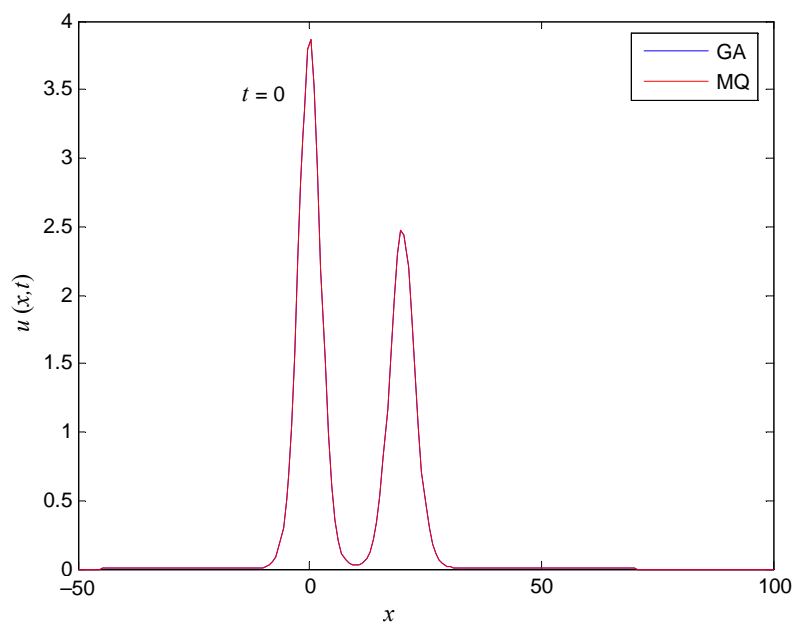

We use multiquadric and Gaussian radial basis functions in our numerical simulations to solve this problem. The spatial domain is selected as $[a, b]=[-30,120]$ with $\Delta x=0.75$. The calculation is carried out up to time $t=$ 50 taking time step $\Delta t=0.001$. The values of the parameters used in above equation are selected as:

$x_{1}=-20, x_{2}=0, x_{3}=20, \sigma=\frac{4}{\sqrt{105}}, A_{1}=\frac{10}{\sigma}, A_{2}=\frac{8}{\sigma}, A_{3}=\frac{6}{\sigma}$

The three solitary waves propagate towards right. The process of interaction is shown in Figure 8. During this process the taller wave moves faster and catches up the smaller waves and then the three waves separate from each other. The shape of the three solitary waves after collision is maintained. From Table 8 we can see that the invariants of motion remain almost conserved as time increases. The variation in the three conserved quantities

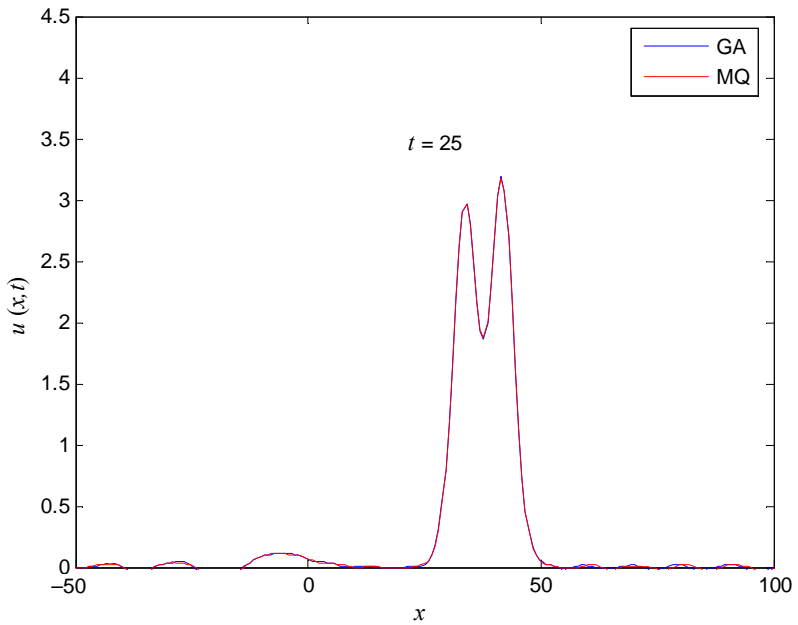

Figure 7. Interaction of two solitons for Kawahara equation Example 3.4.

Table 7. Invariants for interaction of two solitons for Example 3.4.

\begin{tabular}{ccccccc}
\hline \multicolumn{3}{c}{ MQ } & & \multicolumn{2}{c}{ GA } \\
\hline time & $I_{1}$ & $I_{2}$ & $I_{3}$ & $I_{1}$ & $I_{2}$ & $I_{3}$ \\
0 & 40.509259 & 45.836141 & -32.37219 & 40.509259 & 45.836141 & -32.37218 \\
10 & 40.507987 & 45.839240 & -32.12856 & 40.492695 & 45.837775 & -32.12820 \\
20 & 40.499950 & 45.8427481 & -32.73918 & 40.466653 & 45.839353 & -32.73746 \\
30 & 40.5207361 & 45.8359623 & -32.58956 & 40.571742 & 45.840373 & -32.59560 \\
40 & 40.5552400 & 45.8443065 & -32.10125 & 40.579624 & 45.841964 & -32.12278 \\
50 & 40.4838916 & 45.8509384 & -32.15991 & 40.412842 & 45.843648 & -32.14082 \\
& & & & & & \\
\hline
\end{tabular}

is found to be in the range:

$$
\begin{aligned}
& \text { MQ } 51.47263 \leq I_{1} \leq 51.56859,51.10049 \leq I_{2} \leq 51.15838, \\
& -33.63806 \leq I_{3} \leq-33.06973, \\
& \text { GA } 51.47263 \leq I_{1} \leq 51.47389,51.10049 \leq I_{2} \leq 51.10452, \\
& -33.63806 \leq I_{3} \leq-33.39361 .
\end{aligned}
$$

\section{Example 3.6}

In this example we will show the phenomena of wave generation for Equation (1). We consider the following initial condition

$$
u(x, 0)=\beta \operatorname{sech}^{4}\left(\frac{1}{2 \sqrt{13}}\left(x-x_{o}\right)\right)
$$

Computational domain $[-40,130]$ with $h=0.625$ is considered. The scheme is run up to time $t=18$. We take 
$\beta=10$. The single solitary wave splits in to three solitary waves. The conserved quantities, height and position of three waves are calculated at various time levels as shown in Table 9. With passage of time the leading wave owing to its faster velocity gets far from the other two waves as shown in Figure 9. Variation in three conserved quantities is found to be in the following range:

Table 8. Invariants for interaction of three solitons for Example 3.5.

\begin{tabular}{ccccccc}
\hline \multirow{2}{*}{ time } & \multicolumn{3}{c}{ MQ } & \multicolumn{2}{c}{ GA } \\
\cline { 2 - 6 } & $I_{1}$ & $I_{2}$ & $I_{3}$ & $I_{1}$ & $I_{2}$ & $I_{3}$ \\
\hline 0 & 51.472631 & 51.100493 & -33.63806 & 51.472631 & 51.100493 & -33.63806 \\
10 & 51.631982 & 51.094986 & -33.40011 & 51.835962 & 51.098240 & -33.39015 \\
20 & 51.688762 & 51.103862 & -34.23846 & 51.848746 & 51.099504 & -34.21111 \\
30 & 51.664151 & 51.127187 & -35.19047 & 51.690220 & 51.101129 & -35.15871 \\
40 & 51.601572 & 51.14098 & -33.85613 & 51.493752 & 51.102653 & -33.83892 \\
50 & 51.568591 & 51.15838 & -33.06973 & 51.473898 & 51.104529 & -33.39361 \\
\hline
\end{tabular}

Table 9. Invariants for interaction of three solitons for Example 3.6.

\begin{tabular}{|c|c|c|c|c|c|c|}
\hline \multirow{2}{*}{ time } & \multicolumn{2}{|c|}{ Leading wave } & \multicolumn{2}{|c|}{ Second wave } & \multicolumn{2}{|c|}{ Third wave } \\
\hline & Height & Position & Height & Position & Height & Position \\
\hline \multicolumn{7}{|l|}{ MQ } \\
\hline 0 & 10 & 0 & - & - & - & - \\
\hline 2 & 13.990228 & 12.5 & 7.983753 & 5.625 & 2.964111 & -0.625 \\
\hline 4 & 13.645838 & 22.5 & 8.471020 & 11.875 & 3.247767 & 1.875 \\
\hline 8 & 14.024270 & 43.125 & 8.34795 & 24.375 & 3.427390 & 7.5 \\
\hline 12 & 13.93545 & 63.75 & 8.48316 & 36.25 & 3.49294 & 12.5 \\
\hline 18 & 13.90723 & 94.375 & 8.34579 & 55 & 3.50220 & 20 \\
\hline
\end{tabular}
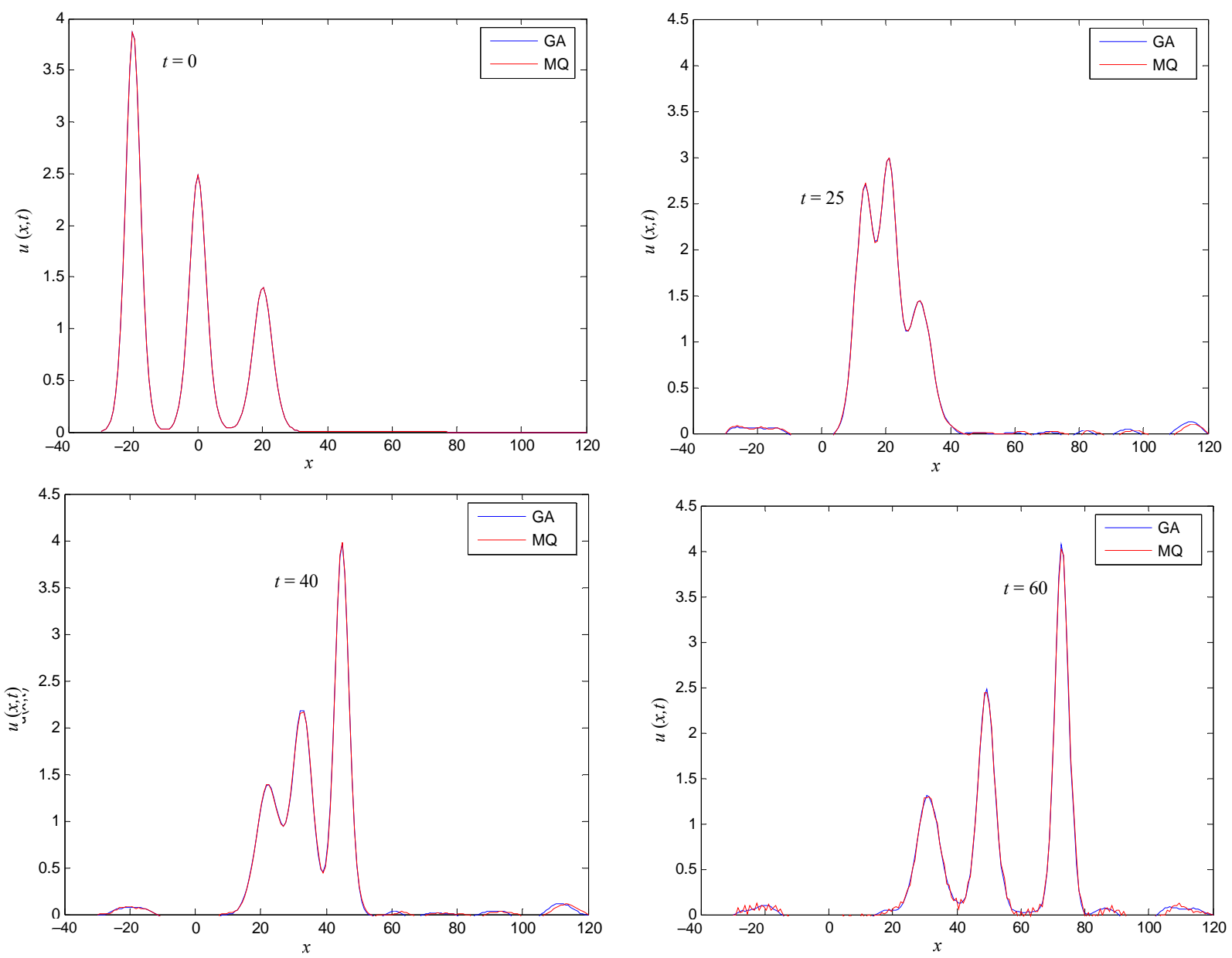

Figure 8. Interaction of three solitons for Example 3.5. 

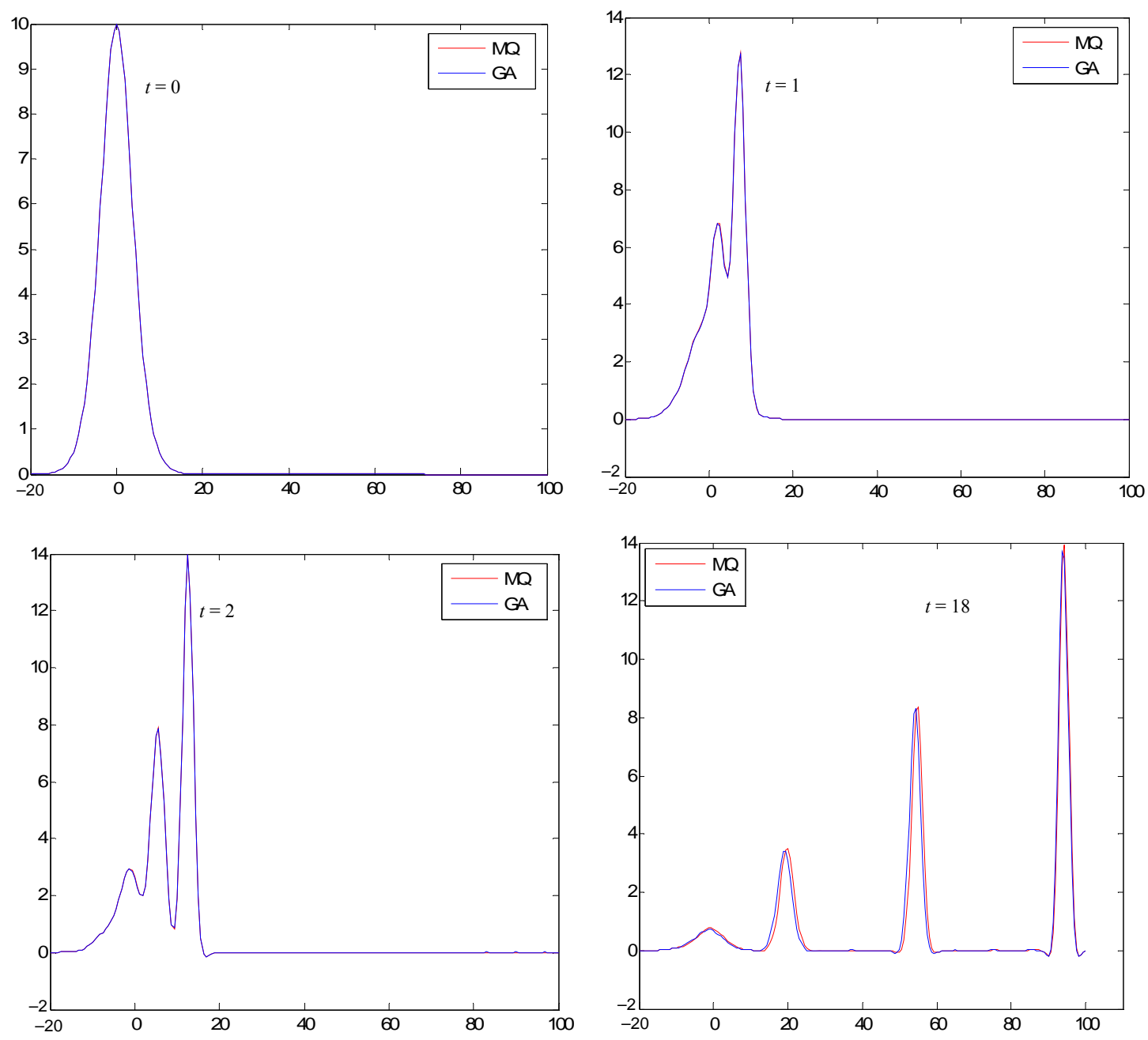

Figure 9. Generation of waves for Example 3.6.

MQ $96.14365 \leq I_{1} \leq 96.14365,329.65039 \leq I_{2}$ $\leq 329.65039,-875.41604 \leq I_{3} \leq-701.42681$, GA $96.14365 \leq I_{1} \leq 96.14365,329.65039 \leq I_{2}$ $\leq 329.65039,-875.41612 \leq I_{3} \leq-706.85789$.

\section{Conclusions}

In this paper, we have used method of lines coupled with radial basis functions for numerical solution of Kawahara type equations. The numerical results describing motion of single solitary wave, interaction of two and three solitary waves and phenomena of wave generation have been discussed. The accuracy of the solution depends upon the choice of the shape parameter, which has been selected experimentally. The numerical results using MQ and GA for Kawahara equation are better than the Crank Nicolson differential quadrature algorithm [10]. The invariants of motion remained conserved during the proc- ess of computation for all cases. Two main advantages of this method are mesh less property and use of ODE solvers of high quality and their codes to approach the solution of PDEs. Also the presented method is simple, easy to implement because no mesh is required in the problem domain. Only radial distance between the nodes is used to approximate the solution.

\section{Acknowledgements}

The first author is thankful to HEC Pakistan for financial help through Grant No. 063-112367-Ps3-096. We are also thankful to the reviewers for their constructive comments.

\section{References}

[1] T. Kawahara, "Oscillatory Solitary Waves in Dispersive Media," Journal of the Physical Society of Japan, Vol. 33, 
No. 1, 1972, pp. 260-264. doi:10.1143/JPSJ.33.260

[2] T. Bridges and G. Derks, "Linear Instability of Solitary Wave Solutions of the Kawahara Equation and its Generalizations," Society for Industrial and Applied Mathematics: Journal on Mathematical Analysis, Vol. 33, No. 6, 2002, pp. 1356-1378. doi:10.1137/S0036141099361494

[3] J. K. Hunter and J. Scheurle, "Existence of Perturbed Solitary Wave Solutions to a Model Equation for Water Waves," Physica D, Vol. 32, No. 2, 1988, pp. 253-268. doi:10.1016/0167-2789(88)90054-1

[4] D. J. Benny, "Long Waves on Liquid Film," Journal of Mathematical Physics, Vol. 45, 1966, pp. 150-155.

[5] S .P. Lin, "Finite Amplitude Side-Band Stability of a Viscous Film," Journal of Fluid Mechanics, Vol. 63, No. 3, 1974, pp. 417-429. doi:10.1017/S0022112074001704

[6] E. Yusufoglu and A. Bekir, "Symbolic Computation and New Families of Exact Travelling Solutions for the Kawahara and Modified Kawahara Equations," Computers and Mathematics with Applications, Vol. 55, No. 6, 2008, pp. 1113-1121. doi:10.1016/j.camwa.2007.06.018

[7] D. Kaya, "An Explicit and Numerical Solutions of Some Fifth-Order KdV Equation by Decomposition Method," Applied Mathematics and Computation, Vol. 144, No. 2-3, 2003, pp. 353-363.

doi:10.1016/S0096-3003(02)00412-5

[8] E. Yusufoglu, A. Bekir and M. Alp, "Periodic and Solitary Wave Solutions of Kawahara and Modified Kawahara Equations by Sine-Cosine Method," Chaos, Solitons and Fractals, Vol. 37, No. 4, 2008, pp. 1193-1197.

[9] L. Jin, “Application of Variational Iteration Method and Homotopy Perturbation Method to the Modified Kawahara Equation," Mathematical and Computer Modeling, Vol. 49, No. 3-4, 2009, pp. 573-578. doi:10.1016/j.mcm.2008.06.017

[10] A. Korkmaz and I. Dag, "Crank-Nicolson-Differential Quadrature Algorithms for the Kawahara Equation," Chaos Solitons and Fractals, Vol. 42, No. 1, 2009, pp. 65-73. doi:10.1016/j.chaos.2008.10.033

[11] K. Djidjeli, W. G. Price, E. H. Twizell and Y. Wang, "Numerical Methods for the Solution of the Third and Fifth-Order Dispersive Korteweg de Vries Equations," Journal of Computational and Applied Mathematics, Vol, 58, No. 3, 1995, pp. 307-336. doi:10.1016/0377-0427(94)00005-L

[12] J. M. Yuan, J. Shen, W. Jiahong, “A Dual Petrov-Galerkin Method for the Kawahara-Type Equations," Journal of Scientific Computing, Vol. 34, No. 1, 2008, pp. 48-63. doi:10.1007/s10915-007-9158-4

[13] S. Haq and M. Uddin, "RBFs Approximation Method for Kawahara Equation," Engineering Analysis with Boundary Elements, Vol. 35, No. 3, 2011, pp.575-580. doi:10.1016/j.enganabound.2010.07.009

[14] Q. Shen, “A Meshless Method of Lines for the Numerical Solution of KdV Equation Using Radial Basis Functions," Engineering Analysis with Boundary Elements, Vol. 33, No. 10, 2009, pp. 1171-1180. doi:10.1016/j.enganabound.2009.04.008

[15] S. Haq, N. Bibi, S. I. A. Tirmizi and M. Usman, "Meshless Method of Lines for the Numerical Solution of Generalized Kuramoto-Sivashinsky Equation," Applied Mathematics and Computation, Vol. 217, No. 6, 2010, pp. 2404-2413.

[16] W. E. Schiesser, "The Numerical Method of Lines: Integration of Partial Differential Equations," 1st Edition, Academic Press; San Diego California, 1991.

[17] E. J. Kansa, "Multiquadrics a Scattered Data Approximation Scheme with Application to Computational Fluid Dynamics-I, "Computers and Mathematics with Applications, Vol. 19, No. 8-9, 1990, pp. 127-145. doi:10.1016/0898-1221(90)90270-T

[18] E. J. Kansa, "Multiquadrics a Scattered Data Approximation Scheme with Application to Computational Fluid Dynamics-II," Computers and MaFFthematics with Applications, Vol. 19, No. 8-9, 1990, pp.147-161. doi:10.1016/0898-1221(90)90271-K

[19] B. Fornberg, T. A. Driscoll, G. Wright and R. Charles, "Observations on the Behavior of Radial Basis Function Approximations near Boundaries," Computers and Mathematics with Applications, Vol. 43, No. 3-5, 2002, pp. 473-490.

[20] Y. C. Hon and Z. Wu, "A Quasi-Interpolation Method for Solving Stiff Ordinary Differential Equations," International Journal of Numerical Methods for Engineering, Vol. 48, No. 8, 2000, pp. 1187-97.

doi:10.1002/(SICI)1097-0207(20000720)48:8<1187::AI D-NME942>3.0.CO;2-K

[21] C. Franke and R. Schaback, "Solving Partial Differential Equations by Collocation Using Radial Basis Functions," Applied Mathematics and Computation, Vol. 93, No. 1, 1998, pp. 73-82.doi:10.1016/S0096-3003(97)10104-7

[22] A. S. M. Wong, Y. C. Hon, T. S. Li, S. L. Chug and E. J. Kansa, "Multizone Decomposition of Time Dependent Problems Using the Multiquadric Scheme," Computers and Mathematics with Applications, Vol. 37, No. 8, 1999, pp. 23-43. doi:10.1016/S0898-1221(99)00098-X

[23] W. Chen and M. Tanaka, "A Meshless Exponential Convergence, Integration-Free and Boundary-Only RBF Technique," Computers and Mathematics with Applications, Vol. 43, No. 3-5, 2002, pp. 379-391. doi:10.1016/S0898-1221(01)00293-0

[24] G. E. Fasshauer, "Solving Partial Differential Equations by Collocation with Radial Basis Functions," Nashville Vanderbilt University Press, Proceeding of Chamonix, Nashville, 1996.

[25] S. Ul. Islam, A. J. Khattak and I. A. Tirmizi, "A Meshfree Method for Numerical Solution of KdV Equation," Engineering Analysis with Boundary Elements, Vol. 32, No. 10, 2008, pp. 849-855.

[26] A. J. Khattak, S. I. A. Tirmizi and S. Ul. Islam, “Application of Meshfree Collocation Method to a Class of Nonlinear Partial Differential Equations," Engineering Analysis with Boundary Elements, Vol. 33, No. 5, 2009, pp. 661-667. doi:10.1016/j.enganabound.2008.10.001 
[27] R. L. Hardy, "Multiquadric Equations of Topography and other Irregular Surfaces," Journal of Geophysical Research, Vol. 76, No. 8, 1971, pp. 1905-1915. doi:10.1029/JB076i008p01905

[28] C. Franke, "Scattered Data Interpolation: Tests of Some Methods," Mathematics of Computation, Vol. 38, No. 157, 1982, pp. 181-200.

[29] Z. M. Wu, "Solving PDE with Radial Basis Function and the Error Estimation," In: Z. Chen, Y. Li, C. A. Micchelli, Y. Xu and M. Dekker, Ed., Advances in Computational Mathematics, Lecture Notes on Pure and Applied Mathematics, Volume 202, GuangZhou, 1998.

[30] A. E. Tarwater, “A Parameter Study of Hardy's Multiquadric Method for Scattered Data Interpolation," Technical Report, Lawrence Livermore National Laboratory, Livermore, 1985.

[31] M. A. Golberg, C. S. Chen and S. R. Karur, "Improved Multiquadric Approximation for Partial Differential Equations," Engineering Analysis with Boundary Elements, Vol. 18, No. 1, 1996, pp. 9-17. doi:10.1016/S0955-7997(96)00033-1

[32] J. Hickernell, and Y. C. Hon, "Radial Basis Function Approximation of the Surface Wind Field from Scattered Data," International Journal of Applied Science and Computations, Vol. 4, No. 3, 1998, pp. 221-247.

[33] A. S. M. Wong, Y. C. Hon, T. S. Li, S. L. Chug and E. J. Kansa, "Multizone Decomposition of Time Dependent Problems Using the Multiquadric Scheme," Computers and Mathematics with Applications, Vol. 37, No. 8, 1999, pp. 23-43. doi:10.1016/S0898-1221(99)00098-X

[34] L. Ling and E. J. Kansa, "A Least-Square Preconditioner for Radial Basis Functions Collocation Methods," $A d$ vances in Computational Mathematics, Vol 23, No. 1-2, 2005, pp. 31-54. doi:10.1007/s10444-004-1809-5

[35] H. Wendland, "Piecewise Polynomial, Positive Definite and Compactly Supported Radial Functions of Minimal Degree," Advances in Computational Mathematics, Vol. 4, No. 1, 1995, pp. 389-96. doi:10.1007/BF02123482

[36] Y. L. Wu and G. R. Liu, "A Meshfree Formulation of Local Radial Point Interpolation Method (LRPIM) for Incompressible Flow Simulation," Computational Mechanics, Vol. 30, No. 5-6, 2003, pp. 355-365.

[37] C. K. Lee, X. Liu and S. C. Fan, "Local Multiquadric Approximation for Solving Boundary Value Problems," Computational Mechanics, Vol. 30, No. 5-6, 2003, pp. 396-409. doi:10.1007/s00466-003-0416-5

[38] R. Vertnik and B. Sarler, "Meshless Local Radial Basis Function Collocation Method for Convective-Diffusive Solid-Liquid Phase Change Problems," International Journal of Numerical Methods for Heat \& Fluid flow, Vol. 16, No. 5, 2006, pp. 617-640. doi:10.1108/09615530610669148
[39] M. Buhmann and N. Dyn, "Spectral Convergence of Multiquadric Interpolation," Proceedings of the Edinburgh Mathematical Society, Vol. 36, No. 2, 1993, pp. 319-333. doi:10.1017/S0013091500018411

[40] M. D. Buhmann, "Radial Basis Functions," Cambridge University Press, Cambridge, 2003. doi:10.1017/CBO9780511543241

[41] W. R. Madych and S. A. Nelson, "Error Bounds for Multiquadric Interpolation," In: C. Chui, L. Schumaker and J. Ward Eds., Approximation Theory VI, Academic Press, New York, 1989, pp. 413-416.

[42] W. R. Madych and S. A. Nelson, "Multivariate Interpolation and Conditionally Positive Definite Functions, II," Mathematics of Computation, Vol. 54, No. 189, 1990, pp. 211-230.doi:10.1090/S0025-5718-1990-0993931-7

[43] S. A. Sarra, "Adaptive Radial Basis Function Method for Time Dependent Partial Differential Equations," Applied Numerical Mathematics, Vol. 54, No. 1, 2005, pp. 79-94. doi:10.1016/j.apnum.2004.07.004

[44] G. E. Fasshauer, "On the Numerical Solution of Differential Equations with Radial Basis Functions," Boundary Element Technology XIII, Waterford Institute of Technology Press, Waterford, 1999, pp. 291-300.

[45] L. Collatz, "The Numerical Treatment of Differential Equations," 3rd Edition, Springer, Verlag, 1966.

[46] J. D. Lambert, "Computational Methods in Ordinary Differential Equations", John Wiley and Sons, J. W. Arrowsmith Ltd, Bristol, 1983.

[47] M. K. Jain, "Numerical Solution of Differential Equations," New Age International (P) Ltd., Publishers, New Delhi, 1984.

[48] L. N Trefeten, "Spectral Methods in MATLAB," Society for Industrial and Applied Mathematics, 2000. doi:10.1137/1.9780898719598

[49] R. P. Malik, "On Fifth Order KdV-Type Equation," Bogoliubov laboratory of theoretical physics, Joint Institute for Nuclear Research, Dubna, 1997.

[50] A. M. Wazwaz, "New Solitary Wave Solutions to the Modified Kawahara Equation," Physics Letters A, Vol. 360, No. 4-5, 2007, pp. 588-592. doi:10.1016/j.physleta.2006.08.068

[51] A. M. Wazwaz, "Abundant Solitons Solutions for Several Forms of the Fifth-Order KdV Equation by Using the Tanh Method," Applied Mathematics and Computation, Vol. 182, No. 1, 2006, pp. 283-300. doi:10.1016/j.amc.2006.02.047

[52] J. C. Ceballos, M. Sepulveda and O. P. V. Villagran, "The Korteweg-de Vries-Kawahara Equation in a Bounded Domain and Some Numerical Results," Applied Mathematics and Computation, Vol. 190, No. 1, 2007, pp. 912-936. doi:10.1016/j.amc.2007.01.107 